\title{
Implicit Floquet Analysis of Wind Turbines using Tangent Matrices of a Nonlinear Aeroelastic Code
}

Skjoldan, Peter Fisker; Hansen, Morten Hartvig

Published in:

Wind Energy

Link to article, DOI:

$10.1002 /$ we.467

Publication date:

2012

Link back to DTU Orbit

Citation (APA):

Skjoldan, P. F., \& Hansen, M. H. (2012). Implicit Floquet Analysis of Wind Turbines using Tangent Matrices of a Nonlinear Aeroelastic Code. Wind Energy, 15(2), 275-287. https://doi.org/10.1002/we.467

\section{General rights}

Copyright and moral rights for the publications made accessible in the public portal are retained by the authors and/or other copyright owners and it is a condition of accessing publications that users recognise and abide by the legal requirements associated with these rights.

- Users may download and print one copy of any publication from the public portal for the purpose of private study or research.

- You may not further distribute the material or use it for any profit-making activity or commercial gain

- You may freely distribute the URL identifying the publication in the public portal

If you believe that this document breaches copyright please contact us providing details, and we will remove access to the work immediately and investigate your claim. 


\section{Implicit Floquet analysis of wind turbines using tangent matrices of a nonlinear aeroelastic code}

\author{
P. F. Skjoldan \\ Loads, Aerodynamic and Control \\ Siemens Wind Power A/S \\ Dybendalsvænget 3, DK-2630 Taastrup, Denmark
}

\author{
M. H. Hansen \\ Wind Energy Division, \\ National Laboratory for Sustainable Energy, Ris $\varnothing$ DTU, \\ Frederiksborgvej 399, DK-4000 Roskilde, Denmark
}

\begin{abstract}
The aeroelastic code BHawC for calculation of the dynamic response of a wind turbine uses a nonlinear finite element formulation. Most wind turbine stability tools for calculation of the aeroelastic modes are, however, based on separate linearised models. This paper presents an approach to modal analysis where the linear structural model is extracted directly from BHawC using the tangent system matrices when the turbine is in a steady state. A purely structural modal analysis of the periodic system for an isotropic rotor operating at a stationary steady state is performed by eigenvalue analysis after describing the rotor degrees of freedom in the inertial frame with the Coleman transformation. For general anisotropic systems implicit Floquet analysis, which is less computationally intensive than classical Floquet analysis, is used to extract the least damped modes.

Both methods are applied to a model of a three-bladed 2.3 MW Siemens wind turbine. Frequencies match individually and with a modal identification on time simulations with the nonlinear model. The implicit Floquet analysis performed for operation in a periodic steady state shows that the response of a single mode contains multiple harmonic components differing in frequency by the rotor speed.
\end{abstract}

Keywords: modal analysis, Floquet analysis, rotor dynamics

\section{Introduction}

Today, advanced nonlinear finite element codes $[1,2,3]$ are routinely used for load calculations on wind turbines. Most wind turbine stability tools for calculation of the aeroelastic modes are, however, based on separate linearised models. Stability analysis can be divided into three steps: First a calculation of the steady state, then a linearisation of the equations of motion about the steady state, and last a modal analysis to extract modal frequencies, damping, and mode shapes. This paper presents an approach to linearised structural modal analysis based directly on the nonlinear wind turbine aeroelastic code BHawC [3] applicable to any periodic steady state.

The equations of motion for a wind turbine operating at a constant mean rotor speed contain periodic coefficients, preventing direct eigenvalue analysis of the system. Most recent wind turbine stability tools $[4,5,6,7]$ incorporate the Coleman transformation, also known as the multiblade coordinate transformation, which describes the rotor degrees of freedom in the inertial frame. This transformation eliminates the periodic coefficients if the rotor is isotropic, i.e., consists of identical symmetrically mounted blades, and the environment conditions are symmetric. Floquet analysis is, however, applicable to anisotropic rotors and any periodic steady state. It requires integration of the equations of motion over a period of rotor rotation, as many times as there are state variables in the system. Due to the computational burden of this approach it has only been applied to reduced or simplified wind turbine models with a limited number of degrees of freedom $[8,9,10]$. One way to reduce the computation time is Fast Floquet Theory [11] where only one third of the integrations are necessary for a three-bladed isotropic rotor. Another way is to use implicit Floquet analysis [12] where the least damped modes can be extracted after a limited number of integrations. 
Stol et al. [13] compare the Coleman transformation approach applied to a periodic steady state, where the remaining periodic coefficients are averaged away, with Floquet analysis and finds small differences in modal frequencies and damping, concluding that it is not necessary to use Floquet analysis.

Another approach to modal analysis is system identification $[14,15,16]$ which operates on the response from numerical simulations or measurements, and no knowledge of the system equations is needed to extract the modal properties. The accuracy of the methods is, however, limited and depends on the chosen excitation.

In this paper, tangent matrices for mass, damping, and stiffness are extracted from the aeroelastic code BHawC. If the rotor is isotropic and the steady state is stationary, the Coleman transformation is applied before extracting the modal parameters by eigenvalue analysis. For an anisotropic system, implicit Floquet analysis is used for the modal analysis. When the system is isotropic the response of a single mode contains a single harmonic component for tower degrees of freedom and up to three components for the blades. The response of a single mode in the anisotropic system on both blades and tower contains multiple harmonic components differing in frequency by the rotor speed.

Section 2 of this paper describes the BHawC model and Section 3 explains the methods for modal analysis, the Coleman transformation approach, implicit Floquet analysis and also partial Floquet analysis, a system identification technique. In Section 4 the methods are applied to model of a wind turbine. Section 5 discusses the approaches and Section 6 concludes the paper.

\section{Structural model}

The BHawC wind turbine aeroelastic code [3] is based on a structural finite element model sketched in Figure 1, where the main structural parts, tower, nacelle, shaft, hub, and blades, are modelled as two-node 12-degrees of freedom Timoshenko beam elements. The code uses a corotational formulation, where each element has its own coordinate system that rotates with the element. The elastic deformation is described in the element frame, while the movement of the element coordinate system accounts for rigid body motion. In this way, a geometrically nonlinear model is obtained using linear finite elements.

The configuration of the system, defined by nodal positions $\mathbf{p}$ and orientations $\mathbf{q}$, nodal velocities $\dot{\mathbf{u}}$ (of both positions and orientations) and nodal accelerations ü must satisfy the equilibrium equation

$-\mathbf{f}_{\text {iner }}(\mathbf{p}, \mathbf{q}, \dot{\mathbf{u}}, \ddot{\mathbf{u}})-\mathbf{f}_{\text {damp }}(\mathbf{q}, \dot{\mathbf{u}})+\mathbf{f}_{\text {int }}(\mathbf{p}, \mathbf{q})=\mathbf{f}_{\text {ext }}$

where $\mathbf{f}_{\text {iner }}, \mathbf{f}_{\text {damp }}, \mathbf{f}_{\text {int }}$, and $\mathbf{f}_{\text {ext }}$ are the inertial, damping, internal, and external force vectors, respectively, and $(\dot{)}=\mathrm{d} / \mathrm{d} t$ denotes a time derivative. To find this equilibrium configuration, increments of the positions and orientations $\delta \mathbf{u}$, velocities $\delta \dot{\mathbf{u}}$ and accelerations $\delta \ddot{\mathbf{u}}$ are found by Newton-Raphson iteration with the tangent relation obtained from the variation of Equation (1) as

$$
\mathbf{M}(\mathbf{q}) \delta \ddot{\mathbf{u}}+\mathbf{C}(\mathbf{q}, \dot{\mathbf{u}}) \delta \dot{\mathbf{u}}+\mathbf{K}(\mathbf{p}, \mathbf{q}, \dot{\mathbf{u}}, \ddot{\mathbf{u}}) \delta \mathbf{u}=\mathbf{r}
$$

where $\mathbf{M}, \mathbf{C}$, and $\mathbf{K}$ are the tangent mass, damping/gyroscopic, and stiffness matrices, respectively, and $\mathbf{r}=\mathbf{f}_{\text {ext }}+\mathbf{f}_{\text {iner }}+\mathbf{f}_{\text {damp }}-\mathbf{f}_{\text {int }}$ is the residual. The stiffness matrix is composed of constitutive, geometric, and inertial stiffness. The orientation of the nodes $\mathbf{q}$ is described by quaternions [17], a general four-parameter representation equivalent to a triad, which for node number $i$ is updated as

$$
\mathbf{q}_{i}:=\mathbf{q}_{i} \otimes \operatorname{quat}\left(\delta \mathbf{u}_{i, \mathrm{rot}}\right)
$$

where $\delta \mathbf{u}_{i, \text { rot }}$ contains three rotations that are assumed infinitesimal and thus commute, and where this rotation vector is transformed by the function termed quat into a quaternion, which is

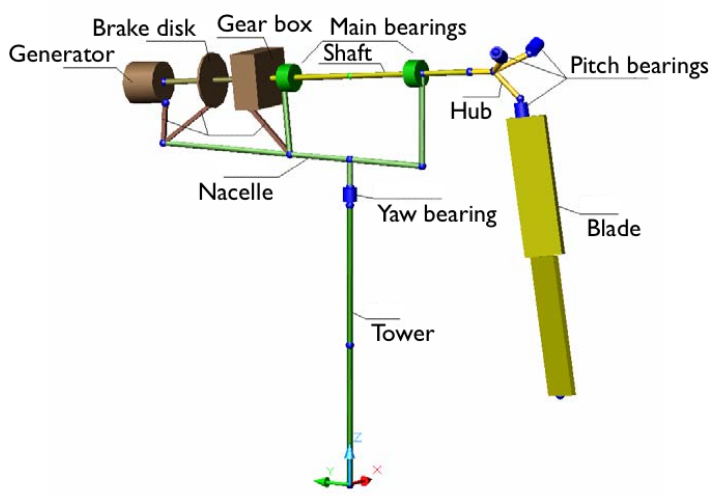

Figure 1: Sketch of BHawC model substructures. 
used to update the nodal quaternion $\mathbf{q}_{i}$ employing the special quaternion product indicated by $\otimes$ which maintains the unity of the quaternion. The nodal positions $\mathbf{p}$ and nodal velocities $\dot{\mathbf{u}}$ and accelerations $\ddot{\mathbf{u}}$ are updated by regular addition of the positional part of $\delta \mathbf{u}, \delta \dot{\mathbf{u}}$ and $\delta \ddot{\mathbf{u}}$, respectively. All components in $\mathbf{p}, \mathbf{q}$, and $\delta \mathbf{u}$ are absolute and described in a global frame.

The present work considers small perturbations in position and orientation $\mathbf{y}$, velocity $\dot{\mathbf{y}}$, and acceleration $\ddot{\mathbf{y}}$ to a steady state operation at constant mean rotor speed $\Omega$ defined by $\left(\mathbf{p}_{\mathrm{ss}}, \mathbf{q}_{\mathrm{ss}}, \dot{\mathbf{u}}_{\mathrm{ss}}, \ddot{\mathbf{u}}_{\mathrm{ss}}\right)$, the steady state positions, orientations, velocities, and accelerations, respectively, all periodic with the rotor period $T=$ $2 \pi / \Omega$. The linearised equations of motion are obtained from Equation (2) at $\mathbf{r} \approx \mathbf{0}$ as

$$
\begin{aligned}
\mathbf{M}\left(\mathbf{q}_{\mathrm{ss}}\right) \ddot{\mathbf{y}}+ & \mathbf{C}\left(\mathbf{q}_{\mathrm{ss}}, \dot{\mathbf{u}}_{\mathrm{ss}}\right) \dot{\mathbf{y}} \\
& +\mathbf{K}\left(\mathbf{p}_{\mathrm{ss}}, \mathbf{q}_{\mathrm{ss}}, \dot{\mathbf{u}}_{\mathrm{ss}}, \ddot{\mathbf{u}}_{\mathrm{ss}}\right) \mathbf{y}=\mathbf{0}
\end{aligned}
$$

where the matrices $\mathbf{M}, \mathbf{C}$, and $\mathbf{K}$ are the $T$ periodic tangent system matrices which are employed in the modal analysis described in the next section.

\section{Methods}

In this section four methods for modal analysis of structures with rotors are presented.

\subsection{Coleman approach}

The Coleman transformation requires identical degrees of freedom on each blade, and therefore the equations of motion (4) in global coordinates are first transformed into substructure coordinates $\mathbf{y}_{\mathrm{T}}$. The transformation is

$$
\begin{aligned}
& \mathbf{y}=\mathbf{T} \mathbf{y}_{\mathrm{T}} \\
& \mathbf{T}=\operatorname{diag}\left(\mathbf{I}_{N_{\mathrm{s}}}, \mathbf{T}_{\mathrm{r}}, \mathbf{T}_{\mathrm{b} 1}, \mathbf{T}_{\mathrm{b} 2}, \mathbf{T}_{\mathrm{b} 3}\right)
\end{aligned}
$$

where $\mathbf{T}$ is a block diagonal time-variant matrix composed of the identity matrix $\mathbf{I}_{N_{\mathrm{s}}}$ sized by the number of degrees of freedom of the tower, nacelle, and drivetrain, $\mathbf{T}_{\mathrm{r}}$ transforming the degrees of freedom on the shaft and hub into a hub centre frame, and $\mathbf{T}_{\mathrm{bj}}$ transforming the degrees of freedom on blade number $j=1,2,3$ into a local frame for blade $j$. The triads are obtained in the periodic steady state, and thus $\mathbf{T}$ is $T$-periodic.
The time-variant transformation into inertial frame coordinates $\mathbf{z}$ is

$$
\begin{aligned}
\mathbf{y}_{\mathrm{T}} & =\mathbf{B} \mathbf{z} \\
\mathbf{B} & =\operatorname{diag}\left(\mathbf{I}_{N_{\mathrm{s}}}, \mathbf{B}_{\mathrm{r}}, \mathbf{B}_{\mathrm{b}}\right)
\end{aligned}
$$

where $\mathbf{B}_{\mathrm{r}}$ is a simple rotational transformation of the shaft and hub, and $\mathbf{B}_{\mathrm{b}}$ is the Coleman transformation introducing multi-blade coordinates for a three-bladed rotor $[11,18]$ as

$$
\mathbf{B}_{\mathrm{b}}=\left[\begin{array}{lll}
\mathbf{I}_{N_{\mathrm{b}}} & \mathbf{I}_{N_{\mathrm{b}}} \cos \psi_{1} & \mathbf{I}_{N_{\mathrm{b}}} \sin \psi_{1} \\
\mathbf{I}_{N_{\mathrm{b}}} & \mathbf{I}_{N_{\mathrm{b}}} \cos \psi_{2} & \mathbf{I}_{N_{\mathrm{b}}} \sin \psi_{2} \\
\mathbf{I}_{N_{\mathrm{b}}} & \mathbf{I}_{N_{\mathrm{b}}} \cos \psi_{3} & \mathbf{I}_{N_{\mathrm{b}}} \sin \psi_{3}
\end{array}\right]
$$

where $\psi_{j}=\Omega t+2 \pi(j-1) / 3$ is the mean azimuth angle to blade number $j$, and $N_{\mathrm{b}}$ is the number of degrees of freedom on each blade. The inertial frame coordinate vector

$$
\mathbf{z}=\left\{\mathbf{y}_{\mathrm{s}}^{\mathrm{T}} \mathbf{z}_{\mathrm{r}}^{\mathrm{T}} \mathbf{a}_{0}^{\mathrm{T}} \mathbf{a}_{1}^{\mathrm{T}} \mathbf{b}_{1}^{\mathrm{T}}\right\}^{\mathrm{T}}
$$

contains the untransformed coordinates for tower, nacelle, and drivetrain $\mathbf{y}_{\mathrm{s}}$, the coordinates for shaft and hub $\mathbf{z}_{\mathrm{r}}$ measured in a non-rotating frame aligned with the hub, and the multi-blade symmetric coordinates $\mathbf{a}_{0}$, cosine coordinates $\mathbf{a}_{1}$ and sine coordinates $\mathbf{b}_{1}$. Details on how multiblade coordinates describe the motion of a wind turbine rotor in the inertial frame are discussed in $[19,20]$.

The Coleman transformed equations are obtained by first inserting Equation (5) into (4), then converting to first order form and last introducing the inertial frame transformation in Equation (6) as $\dot{\mathbf{z}}_{2}=\operatorname{diag}(\mathbf{B}, \mathbf{B}) \mathbf{z}_{2}$ where $\mathbf{z}_{2}=$ $\left\{\mathbf{z}^{\mathrm{T}} \tilde{\mathbf{z}}^{\mathrm{T}}\right\}^{\mathrm{T}}$ is the state vector with $\tilde{\mathbf{z}}=\dot{\mathbf{z}}+\overline{\boldsymbol{\omega}} \mathbf{z}$ and the constant matrix $\overline{\boldsymbol{\omega}}=\mathbf{B}^{-1} \dot{\mathbf{B}}$. The result is

$$
\begin{aligned}
\dot{\mathbf{z}}_{2} & =\mathbf{A}_{\mathrm{B}} \mathbf{z}_{2} \\
\mathbf{A}_{\mathrm{B}} & =\left[\begin{array}{cc}
-\overline{\boldsymbol{\omega}} & \mathbf{I} \\
-\mathbf{M}_{\mathrm{B}}^{-1} \mathbf{K}_{\mathrm{B}} & -\mathbf{M}_{\mathrm{B}}^{-1} \mathbf{C}_{\mathrm{B}}-\overline{\boldsymbol{\omega}}
\end{array}\right]
\end{aligned}
$$

where $\mathbf{A}_{\mathrm{B}}$ is the Coleman transformed system matrix and

$$
\begin{aligned}
\mathbf{M}_{\mathrm{B}} & =\mathbf{B}^{-1} \mathbf{T}^{\mathrm{T}} \mathbf{M} \mathbf{T} \mathbf{B} \\
\mathbf{C}_{\mathrm{B}} & =\mathbf{B}^{-1} \mathbf{T}^{\mathrm{T}}(\mathbf{C} \mathbf{T}+2 \mathbf{M} \dot{\mathbf{T}}) \mathbf{B} \\
\mathbf{K}_{\mathrm{B}} & =\mathbf{B}^{-1} \mathbf{T}^{\mathrm{T}}(\mathbf{K} \mathbf{T}+\mathbf{C} \dot{\mathbf{T}}+\mathbf{M} \ddot{\mathbf{T}}) \mathbf{B}
\end{aligned}
$$

are the Coleman transformed mass, damping/gyroscopic, and stiffness matrices, respectively. If the system is isotropic, then $\mathbf{A}_{\mathrm{B}}$ is timeinvariant and a transient solution of Equation (9) 
is

$$
\mathbf{z}_{2}=\mathrm{e}^{\mathbf{A}_{\mathrm{B}} t} \mathbf{z}_{2}(0)=\mathbf{V} \mathrm{e}^{\boldsymbol{\Lambda} t} \mathbf{q}(0)
$$

where $\boldsymbol{\Lambda}$ is a diagonal matrix containing the eigenvalues of $\mathbf{A}_{\mathrm{B}}, \mathbf{V}$ contains the corresponding eigenvectors as columns and $\mathbf{q}(0)=\mathbf{V}^{-1} \mathbf{z}_{2}(0)$ are the initial conditions in modal coordinates. It is assumed that all eigenvectors are linearly independent.

The blade motion given in the inertial frame in Equation (11) can be transformed back into the rotating frame using Equation (6) as [20]

$$
\begin{aligned}
& y_{\mathrm{T}, \mathrm{ik}}=\mathrm{e}^{\sigma_{k} t}\left(A_{0, i k} \cos \left(\omega_{k} t+\varphi_{0, i k}\right)\right. \\
& +A_{\mathrm{BW}, i k} \cos \left(\left(\omega_{k}+\Omega\right) t+\varphi_{j}+\varphi_{\mathrm{BW}, i k}\right) \\
& \left.+A_{\mathrm{FW}, i k} \cos \left(\left(\omega_{k}-\Omega\right) t-\varphi_{j}+\varphi_{\mathrm{FW}, i k}\right)\right)
\end{aligned}
$$

where $\varphi_{j}=2 \pi(j-1) / 3, \sigma_{k}$ and $\omega_{k}$ are the modal damping and frequency of mode number $k$, respectively, given by the eigenvalue $\lambda_{k}=\sigma_{k}+\mathrm{i} \omega_{k}$ with $\mathrm{i}=\sqrt{-1}$. The amplitudes for degree of freedom number $i$ are determined from the components of the eigenvector $\mathbf{v}_{k}$ given in multi-blade coordinates of Equation (8) as $A_{0, i k}=\left|a_{0, i k}\right|$ and

$$
\begin{aligned}
A_{\mathrm{BW}, i k}= & \frac{1}{2}\left(\left(\operatorname{Re}\left(a_{1, i k}\right)+\operatorname{Im}\left(b_{1, i k}\right)\right)^{2}\right. \\
& \left.+\left(\operatorname{Re}\left(b_{1, i k}\right)-\operatorname{Im}\left(a_{1, i k}\right)\right)^{2}\right)^{1 / 2} \\
A_{\mathrm{FW}, i k}= & \frac{1}{2}\left(\left(\operatorname{Re}\left(a_{1, i k}\right)-\operatorname{Im}\left(b_{1, i k}\right)\right)^{2}\right. \\
& \left.+\left(\operatorname{Re}\left(b_{1, i k}\right)+\operatorname{Im}\left(a_{1, i k}\right)\right)^{2}\right)^{1 / 2}
\end{aligned}
$$

where the subscripts $0, B W$, and FW denote symmetric, backward whirling, and forward whirling motion, respectively.

\subsection{Classical Floquet analysis}

Floquet analysis enables the solution of the periodic equations of motion directly without an explicit transformation. Equation (4) is written in first order form

$$
\begin{aligned}
\dot{\mathbf{y}}_{2} & =\mathbf{A} \mathbf{y}_{2} \\
\mathbf{A} & =\left[\begin{array}{cc}
\mathbf{0} & \mathbf{I} \\
-\mathbf{M}^{-1} \mathbf{K} & -\mathbf{M}^{-1} \mathbf{C}
\end{array}\right]
\end{aligned}
$$

where $\mathbf{y}_{2}=\left\{\mathbf{y}^{\mathrm{T}} \dot{\mathbf{y}}^{\mathrm{T}}\right\}^{\mathrm{T}}$ is the state vector and $\mathbf{A}$ is the $T$-periodic system matrix.

Floquet theory [21] states that the solution to Equation (15) is of the form

$$
\mathbf{y}_{2}=\mathbf{U e}^{\boldsymbol{\Lambda} t} \mathbf{U}^{-1}(0) \mathbf{y}_{2}(0)
$$

where $\mathbf{U}$ is a $T$-periodic matrix and $\boldsymbol{\Lambda}$ is a diagonal matrix. One way to construct this solution is to form a fundamental solution to Equation (15) as

$$
\boldsymbol{\varphi}=\left[\begin{array}{llll}
\varphi_{1} & \varphi_{2} & \ldots & \varphi_{N}
\end{array}\right]
$$

over one period, $t \in[0 ; T]$, where $N$ is the number of state variables, such that $\dot{\varphi}=\mathbf{A} \varphi$. The monodromy matrix defined as

$$
\mathbf{C}=\varphi^{-1}(0) \varphi(T)
$$

contains all modal properties, which can be extracted from the eigenvalue decomposition

$$
\mathbf{C}=\mathbf{V J V}^{-1}
$$

where $\mathbf{V}$ contains the column eigenvectors $\mathbf{v}_{k}$ of $\mathbf{C}$, which are all assumed to be linearly independent, and $\mathbf{J}$ is a diagonal matrix containing the eigenvalues $\rho_{k}$ of $\mathbf{C}$, called the characteristic multipliers. The characteristic exponents $\lambda_{k}=\sigma_{k}+\mathrm{i} \omega_{k}$ contain the frequency $\omega_{k}$ and damping $\sigma_{k}$ and are related to the characteristic multipliers as $\rho_{k}=\exp \left(\lambda_{k} T\right)$. Because the complex logarithm is not unique, the frequency is not determined uniquely, and the principal frequency $\omega_{\mathrm{p}, k}$ and the damping $\sigma_{k}$ are defined from the characteristic multipliers as

$$
\begin{aligned}
\sigma_{k} & =\frac{1}{T} \ln \left(\left|\rho_{k}\right|\right) \\
\omega_{\mathrm{p}, k} & =\frac{1}{T} \arg \left(\rho_{k}\right)
\end{aligned}
$$

where $\left.\left.\arg \left(\rho_{k}\right) \in\right]-\pi ; \pi\right]$ is implied, resulting in $\left.\left.\omega_{\mathrm{p}, k} \in\right]-\Omega / 2 ; \Omega / 2\right]$. Any integer multiple of the rotor speed can be added to the principal frequency to obtain a more physically meaningful frequency $[22,23]$

$$
\omega_{k}=\omega_{\mathrm{p}, k}+j_{k} \Omega
$$

a choice which also affects the periodic modal matrix $\mathbf{U}$ in Equation (16). This matrix $\mathbf{U}$ contains the periodic mode shapes $\mathbf{u}_{k}$ and is given as [23]

$$
\mathbf{u}_{k}=\boldsymbol{\varphi} \mathbf{v}_{k} \mathrm{e}^{-\lambda_{k} t}
$$

where $\lambda_{k}$ is selected using Equation (21) such that $\mathbf{u}_{k}$ is as constant as possible for degrees of freedom measured in the inertial frame.

Introducing the Fourier transform of the periodic mode shape

$$
\mathbf{u}_{k}=\sum_{j=-\infty}^{\infty} \boldsymbol{U}_{j k} \mathrm{e}^{\mathrm{i} j \Omega t}
$$


the transient solution in Equation (16) can be written as a sum of harmonic components

$$
\mathbf{y}_{2}=\sum_{k=1}^{N} \sum_{j=-\infty}^{\infty} \mathcal{U}_{j k} \mathrm{e}^{\left(\sigma_{k}+\mathrm{i}\left(\omega_{k}+j \Omega\right)\right) t} q_{k}(0)
$$

where $\mathbf{q}(0)=\mathbf{U}^{-1}(0) \mathbf{y}_{2}(0)$. Note that Equation (12) is a special case of this expression for $j=-1,0,1$.

\subsection{Implicit Floquet analysis}

The Implicit Floquet method is here described based on the detailed description in [12], which focuses on computation of the characteristic multipliers from the state transition matrix $\mathbf{\Phi}(T, 0)$. It can be defined in classical Floquet theory as

$$
\boldsymbol{\varphi}(T)=\boldsymbol{\Phi}(T, 0) \boldsymbol{\varphi}(0)
$$

Using Equation (18), the relationship between the state transition and monodromy matrices is derived as

$$
\boldsymbol{\Phi}(T, 0)=\boldsymbol{\varphi}(0) \mathbf{C} \boldsymbol{\varphi}^{-1}(0)
$$

showing that $\boldsymbol{\Phi}(T, 0)$ and $\mathbf{C}$ have identical eigenvalues (characteristic multipliers), and their eigenvectors are related as $\mathbf{v}_{k}=\varphi^{-1}(0) \mathbf{w}_{k}$, where $\mathbf{w}_{k}$ are the eigenvectors of $\boldsymbol{\Phi}(T, 0)$.

The key feature of the state transition matrix is that it defines the solution $\mathbf{y}_{2}(T)=\mathbf{\Phi}(T, 0) \mathbf{y}_{2}(0)$ for a time integration of the system equations (15) over one period $T$ with initial conditions $\mathbf{y}_{2}(0)$. Hence, without knowing the state transition matrix, it is possible to obtain the product of it with an arbitrary vector (the initial state vector) by integration of (15) over one period. The Arnoldi algorithm [24] is a method to approximate the eigenvalues and eigenvectors of a matrix, say $\boldsymbol{\Phi}(T, 0)$, using only the matrix multiplication with $\boldsymbol{\Phi}(T, 0)$ to construct an $m$-sized subspace

$$
\mathbf{P}=\left[\begin{array}{llll}
\mathbf{p}_{1} & \mathbf{p}_{2} & \cdots & \mathbf{p}_{m}
\end{array}\right]
$$

that satisfies the orthonormality condition

$$
\mathbf{P}^{\mathrm{T}} \mathbf{P}=\mathbf{I}
$$

and where the eigenvalues $\tilde{\rho}_{k}$ of the subspace projected state transition matrix

$$
\mathbf{H}=\mathbf{P}^{\mathrm{T}} \boldsymbol{\Phi}(T, 0) \mathbf{P}
$$

converge towards the eigenvalues $\rho_{k}$ of $\boldsymbol{\Phi}(T, 0)$ with the largest modulus as the size $m$ of the subspace increases. The subspace eigenvectors $\tilde{\mathbf{w}}_{k}$ of $\mathbf{H}$ projected back to the full state space converge towards the eigenvectors $\mathbf{w}_{k}$ of $\boldsymbol{\Phi}(T, 0)$, i.e., $\mathbf{w}_{k} \approx \mathbf{P} \tilde{\mathbf{w}}_{k}$.

The Arnoldi algorithm proceeds as follows:

$$
\begin{aligned}
& \text { Choose an arbitrary vector } \mathbf{p}_{1} \text { with }\left|\mathbf{p}_{1}\right|=1 \\
& \text { for } n=1,2, \ldots, m \\
& \mathbf{a}:=\boldsymbol{\Phi} \mathbf{p}_{n} \text { (integration of (15) over } t \in[0 ; T] \text { ) } \\
& \mathbf{b}:=\mathbf{a} \\
& \text { for } j=1,2, \ldots, n \\
& h_{j, n}:=\mathbf{p}_{j}^{\mathrm{T}} \mathbf{a} \\
& \mathbf{b}:=\mathbf{b}-h_{j, n} \mathbf{p}_{j} \\
& \text { end } \\
& \text { if } n<m \\
& h_{n+1, n}:=|\mathbf{b}| \\
& \mathbf{p}_{n+1}:=\mathbf{b} / h_{n+1, n} \\
& \text { end } \\
& \mathbf{p}_{n+1}:=\mathbf{p}_{n+1}-\sum_{j=1}^{n}\left(\mathbf{p}_{j}^{\mathrm{T}} \mathbf{p}_{n+1}\right) \mathbf{p}_{j}
\end{aligned}
$$

The last step in the $n$-loop is an explicit reorthogonalisation to eliminate an otherwise progressing skewness of the subspace basis and thereby ensure convergence of the algorithm [12]. Note that $\mathbf{H}$ with components $h_{j, n}, n=$ $1, \ldots, m, j=1, \ldots, n$, is an upper Hessenberg matrix for which there exist efficient eigenvalue solvers. In practice the Arnoldi algorithm is continued until a desired number of eigenvalues $\tilde{\lambda}_{k}$ with largest modulus and their corresponding eigenvectors $\mathbf{P} \tilde{\mathbf{w}}_{k}$ of the state transient matrix $\boldsymbol{\Phi}(T, 0)$ are converged to within a specific tolerance.

To construct the approximations to the periodic mode shapes (22), the $m \times m$ fundamental solution matrix $\tilde{\varphi}$ to the subspace projected system equations is written as

$$
\tilde{\boldsymbol{\varphi}}=\mathbf{P}^{\mathrm{T}}\left[\begin{array}{llll}
\boldsymbol{\varphi}_{1} & \boldsymbol{\varphi}_{2} & \ldots & \boldsymbol{\varphi}_{m}
\end{array}\right]
$$

where $\varphi_{j}$ is the integrated solution over $t \in[0 ; T]$ of the full system (15) for each initial condition $\mathbf{p}_{j}$, whereby $\tilde{\varphi}(0)=\mathbf{I}$ due to (28). The eigenvectors $\tilde{\mathbf{v}}_{k}$ of the subspace projected monodromy matrix $\tilde{\mathbf{C}}=\tilde{\varphi}^{-1}(0) \tilde{\boldsymbol{\varphi}}(T)$ are therefore identical to the eigenvectors $\tilde{\mathbf{w}}_{k}$ of the subspace projected state transition matrix (29). The periodic mode shapes in the subspace are therefore similar to Equation (22) given by

$$
\tilde{\mathbf{u}}_{k}=\tilde{\varphi} \tilde{\mathbf{w}}_{k} \mathrm{e}^{-\tilde{\lambda}_{k} t}
$$


which by projection back into the full state space using $\mathbf{u}_{k}=\mathbf{P} \tilde{\mathbf{u}}_{k}$ yields the approximated periodic mode shapes of the full system

$$
\mathbf{u}_{k} \approx\left[\begin{array}{llll}
\boldsymbol{\varphi}_{1} & \boldsymbol{\varphi}_{2} & \ldots & \boldsymbol{\varphi}_{m}
\end{array}\right] \tilde{\mathbf{w}}_{k} \mathrm{e}^{-\tilde{\lambda}_{k} t}
$$

where $\tilde{\mathbf{w}}_{k}$ and $\tilde{\lambda}_{k}$ are the eigenvectors and characteristic exponents of $\mathbf{H}$, respectively.

\subsection{Partial Floquet analysis}

Partial Floquet analysis [22] is a system identification technique that operates on signals with the free response of the system, thus no knowledge of the system equations is necessary. The signals can be obtained by numerical simulation or from measurements.

Singular value decomposition is used to eliminate noise and extract the frequency and damping of the most dominant modes from a matrix similar to the monodromy matrix assembled from a limited number of signals spanning several periods. The entries in this matrix can only be sampled once per period for periodic systems, which limits the accuracy because the signal damps away, decreasing the signal to noise ratio. Time-invariant systems can, however, be sampled once per time step. Therefore, partial Floquet analysis is combined with Coleman transformation of the signals [25], such that the response resembles that of a time-invariant system. This approach increases the accuracy and the number of modes that can be extracted from a given signal. However, a careful choice of forcing, that excites all modes of interest to a sufficient level, is necessary to extract the desired modes accurately.

\section{Numerical results}

The modal analysis methods described in the previous sections are applied to a $\mathrm{BHawC}$ model of a $2.3 \mathrm{MW}$ wind turbine with three $45 \mathrm{~m}$ blades, hub height $80 \mathrm{~m}$ and nominal speed $16 \mathrm{rpm}$. The model has 381 structural degrees of freedom.

\subsection{Isotropic system}

The turbine is mounted with identical blades and runs in vacuum neglecting gravity forces, so the system is isotropic. The deflection of the blades due to centrifugal forces is therefore constant in the blade frame. The constant steady state is found at a given azimuth position by solving Equation (1) statically, including centrifugal forces from the constant rotor speed. In this way a steady state with no transients is obtained, and the system matrices become exactly periodic.

\subsubsection{Coleman transformation approach}

Because the system is isotropic, a modal analysis can be performed on the Coleman transformed system matrix. The system matrices $\mathbf{M}$, $\mathbf{C}$ and $\mathbf{K}$ from Equation (4) are extracted at a single azimuth angle and combined into the Coleman transformed system matrix of Equation (9) from which the modal frequencies, damping and eigenvectors given in the inertial frame are extracted. The time-invariance of the system matrix is checked by calculation for several azimuth angles.

Figure 2(a) shows the lowest modal frequencies as function of rotor speed where the frequency is normalised with the lowest modal frequency at $0 \mathrm{rpm}$. The modes are named according to their dominant motion determined from the eigenvector and the whirling amplitudes calculated from equations (13) and (14). The mode labels in Figure 2 first contain the index of that particular mode; then ' $T$ ' for tower, ' $F$ ' for blade flapwise, ' $E$ ' for blade edgewise or 'DRV' for drivetrain; then 'LO' for longitudinal, 'LA' for lateral, 'BW' for backward whirling, 'FW' for forward whirling or ' $\mathrm{S}$ ' for symmetric. For comparison, frequencies extracted from time simulations with the nonlinear BHawC model using the partial Floquet method [25] are also shown. The agreement is within $0.4 \%$ except for modes coupling to the drivetrain, i.e., the drivetrain, edgewise, and lateral tower modes, where the discrepancy is up to $2 \%$ at the highest rotor speed, which is caused by a difficulty with keeping the rotor speed exactly constant in the nonlinear simulation due to the energy dissipated in the oscillation.

Figure 2(b) shows the damping as function of rotor speed where the logarithmic decrement is normalised with the value for the first tower longitudinal mode at $0 \mathrm{rpm}$. The agreement in damping between the results from the linear model and the partial Floquet analysis applied to the nonlinear model is within $6 \%$, except for a discrepancy of up to $20 \%$ for modes coupling to the drivetrain. It must be noted that the purely structural damping of the modes is small, and thus a small 


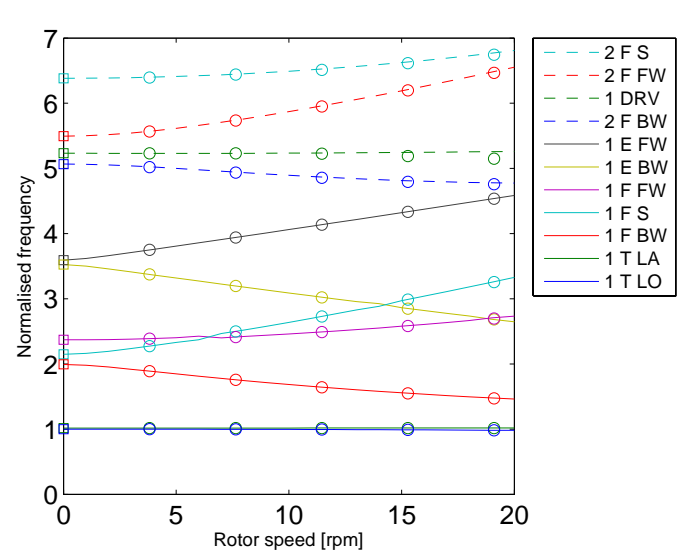

(a)

(b)

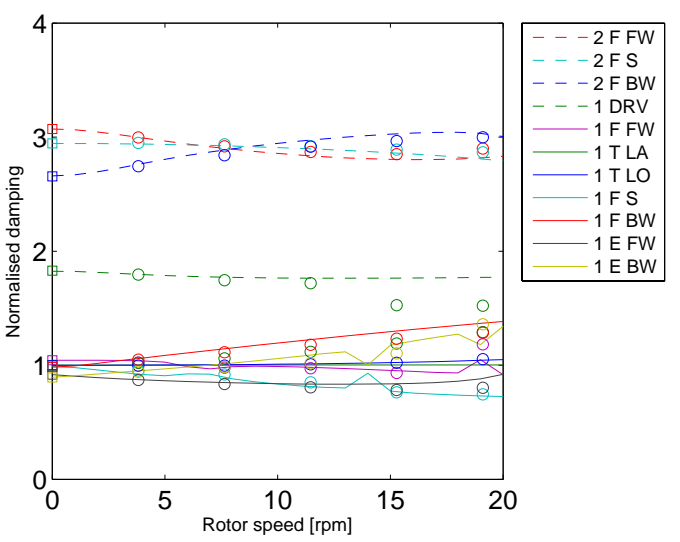

Figure 2: Frequency (a) and damping (b) as function of rotor speed. Standstill eigenvalue analysis (squares), Coleman approach (lines), partial Floquet analysis (circles). Legend entries are ordered after the sequence at $0 \mathrm{rpm}$.

absolute difference leads to a high relative difference. The results also show that damping is more difficult to estimate than frequency using system identification.

\subsubsection{Implicit Floquet analysis}

For the implicit Floquet analysis the system matrices in global coordinates in Equation (4) are extracted from the steady state at 16 azimuth angles equally spaced over a rotor rotation. For interpolation to other azimuth angles a least squares fit of a truncated Fourier series with 8 terms is used. The fundamental solutions in Equation (30) are integrated with a Newmarktype solver from initial conditions determined by the Arnoldi algorithm. The principal frequencies and damping are found from Equation (20) where $\rho_{k}$ are taken as the eigenvalues of the approximated state transition matrix. Figure 3 shows the real part $\sigma$ of the characteristic exponents calculated at each Arnoldi step for a steady state at $12 \mathrm{rpm}$ using a time step of $\Delta t=T / 1024=$ $0.0049 \mathrm{~s}$. The scattering of the highest damping values shows that the highest damped modes are spurious and do not represent actual eigenmodes of the system due to the approximate nature of the implicit Floquet analysis. To exclude these modes from the results, only modes satisfying a strict convergence criterion, where the absolute change of both damping $\sigma_{k}$ and principal frequency $\omega_{\mathrm{p}, k}$ is less than $10^{-10}$ between three successive steps, are retained. After 50 Arnoldi

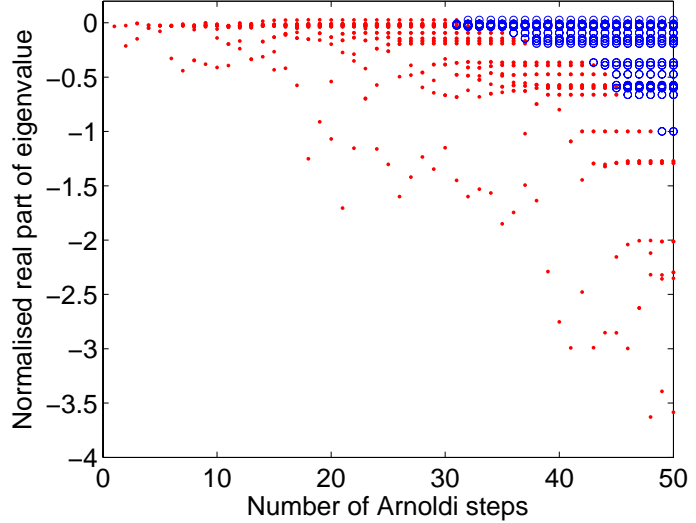

Figure 3: Magnitude of implicit Floquet characteristic multipliers as function of steps in Arnoldi algorithm. - non-converged eigenvalues, o converged eigenvalues.

steps 19 modes are converged. The modal frequencies are determined using Equation (21) by adding $j_{k} \Omega$ to the principal frequency, where $j_{k} \Omega$ is the single non-vanishing harmonic component in a Fourier transform of the periodic mode shape for degrees of freedom on the tower calculated from Equation (32) using the principal frequency $\omega_{\mathrm{p}, k}$. The periodic mode shape components for degrees of freedom on the tower and nacelle calculated with the modal frequency $\omega_{k}$ are thus constant. A detailed description of the process of frequency identification is given in [23].

Figure 4 shows the difference in frequency 


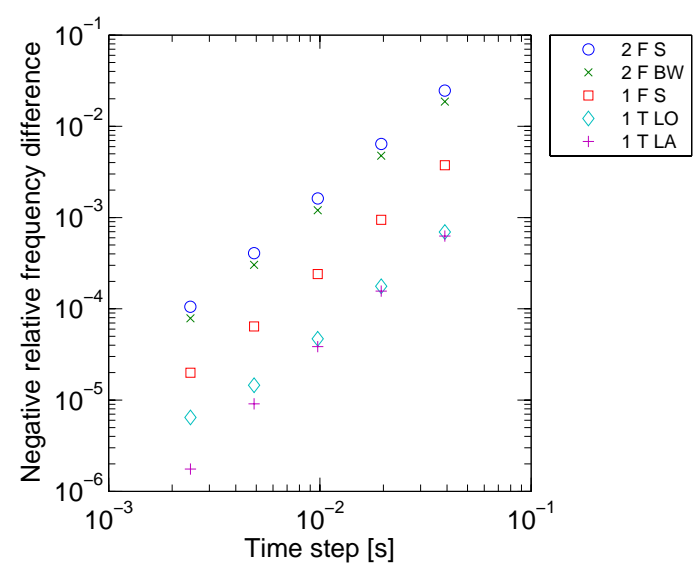

Figure 4: Relative difference in implicit Floquet frequency compared to Coleman approach frequency for selected modes as function of implicit Floquet integration time step.

calculated with the Coleman transformation approach and the implicit Floquet analysis with different integration time steps. The implicit Floquet results converge towards the Coleman transformation results for decreasing time steps, the error being roughly proportional to $\Delta t^{2}$. Predominantly the error increases with the modal frequency. A similar trend is seen for the damping.

Figure 5 shows the dominant harmonic components $\mathcal{U}_{j k}$ in Equation (24) for the first flapwise forward whirling mode shape. The blade mode shape is transformed into substructure coordinates using Equation (5) and contains the rigid body motion of the hub. The zoom factor in the lower right corner indicates how much each component has been enlarged. The ground fixed components in the mode shape are constant, consistent with the solution from the Coleman transformation approach. The mode shape for the blade has harmonic components at $j=-1,0,1$, corresponding to the forward whirling, symmetric, and backward whirling components, respectively, in the Coleman transformation approach. Thus, in a pure excitation of this mode at $12 \mathrm{rpm}$, according to Equation (24) the tower vibrates with the normalised modal frequency $\omega^{\prime}=2.8$, and the blades dominantly vibrate with $\omega^{\prime}-\Omega^{\prime}=2.2$ (FW), and to a lesser extent with $\omega^{\prime}+\Omega^{\prime}=3.3$ (BW) and $\omega^{\prime}=2.8(\mathrm{~S})$ (see Figure 2(a)).
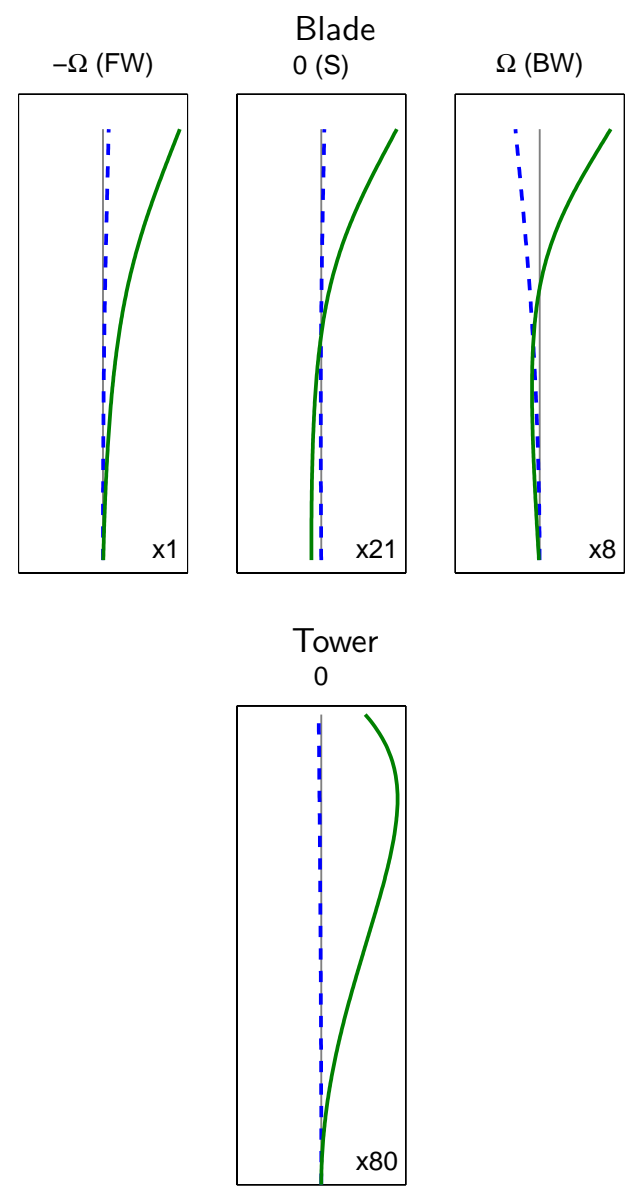

Figure 5: Amplitudes of harmonic components of the first flapwise forward whirling periodic mode shape for the isotropic rotor. Blades (top) — flapwise and - - edgewise, and tower (bottom) - longitudinal and - - lateral.

\subsection{Anisotropic system}

To investigate the effects of an anisotropic rotor on the modal properties, a mass of $485 \mathrm{~kg}$ due to ice coverage defined by DIN-1055-5 [26] is added along the length of blade 1. Figure 6 shows the resulting steady state when running the turbine at $16 \mathrm{rpm}$ with a $10 \mathrm{~m} / \mathrm{s}$ uniform wind field perpendicular to the rotor plane. Note that the wind is used only to drive the rotor and the modal analysis is still purely structural. The steady state varies periodically both for the tower and the blades, and the blade motion for blade 1 is different from that of blades 2 and 3 . The steady state is determined from a time simulation until transients have damped away and system matrices are then extracted at each time step of the 

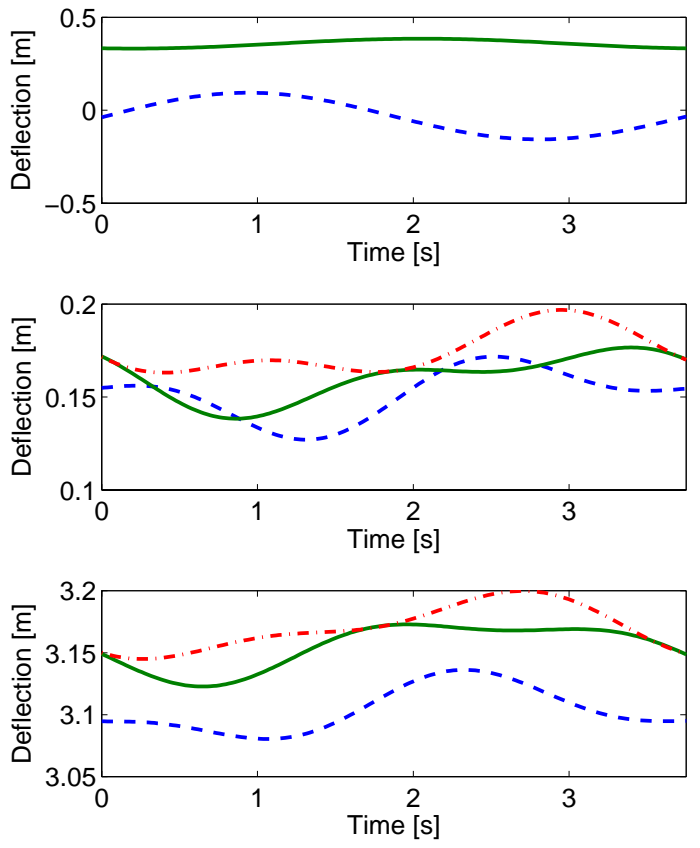

Figure 6: Steady state over one rotor period for the anisotropic rotor at $16 \mathrm{rpm}$. Tower top (top) - longitudinal and - - lateral, blade tips edgewise (middle) - - 1, -2 and $-\cdot-3$, and blade tips flapwise (bottom) - - 1, - 2 and -.- 3 .

steady state simulation and interpolated onto integration time points using a truncated Fourier series with 8 terms. The implicit Floquet analysis is carried out with an integration time step of $T / 1024=0.0037 \mathrm{~s}$ as described for the isotropic case. The frequencies are up to $4 \%$ lower than in the isotropic case due to the added mass on one blade. The change in damping is slightly more pronounced, up to a $17 \%$ decrease for the second flapwise forward whirling mode.

Figure 7 shows the harmonic components $\mathcal{U}_{j k}$ with frequencies $j \Omega$ of the first flapwise forward whirling mode shape for the tower and blade 1 . The tower mode shape now has several harmonic components compared to only one in the isotropic case. The component at $j=0$ is similar in shape to the corresponding one for the isotropic case, but now the dominant component is at $j=-2$, and there is also a significant component at $j=$ -1 .

For the mode shape of blade 1 the harmonic components at $j=-1,0,1$ are similar to the corresponding ones in the isotropic case. However, now the amplitude of the dominant flapwise component at $j=-1$ for blade 1 is three times as high as for blades 2 and 3, and blades 2 and 3 move close to in-phase and in counter-phase with blade 1, as shown in Figure 8 . Thus, in a pure excitation of this mode the tower now vibrates dominantly with the normalised frequency $\omega^{\prime}-2 \Omega^{\prime}=1.6$ in addition to the component at $\omega^{\prime}=2.8$. Blade 1 vibrates dominantly at $\omega^{\prime}-\Omega^{\prime}=2.2$ as for the isotropic case and notably at $\omega^{\prime}-2 \Omega^{\prime}=1.6, \omega^{\prime}-3 \Omega^{\prime}=1.0$ and $\omega^{\prime}+3 \Omega^{\prime}=4.5$ in addition to $\omega^{\prime}+\Omega^{\prime}=3.3$ and $\omega^{\prime}=2.8$ as for the isotropic case.

The identification of the first flapwise forward whirling modal frequency is not done by making the tower mode shape as constant as possible, as in the isotropic case. Rather, the modal frequency is chosen to be close to the one for the similar mode in the isotropic case. A more suitable criterion to give this result is to require that the mode shape with the rotor degrees of freedom in multi-blade coordinates be as constant as possible [27].

The rotor with one ice-covered blade is an example of how an isotropic rotor can change the modal dynamics of the system. Other influences that could cause a similar behaviour is rotor stiffness unbalance, gravity loads, yaw error, and wind shear. A two-bladed rotor is inherently anisotropic and requires a general approach like Floquet analysis.

\section{Discussion}

This paper has presented several different methods for structural modal analysis of wind turbines. The Coleman approach is simple and fast, and its basis in a physical coordinate transformation means that the results are easily interpreted. Its speed makes it useful for doing parameter studies early in the design process. But it is only applicable to isotropic systems. Floquet analysis can be applied to examine special cases where anisotropic effects are suspected to change the modal parameters. The implicit Floquet analysis is an efficient implementation of Floquet analysis for systems with many degrees of freedom. In the example given, the most important modes are extracted after 50 integrations of the system over a rotor period, whereas 762 integrations would be needed for a classical Floquet analysis. Finally, the partial Floquet analysis, or another means of 


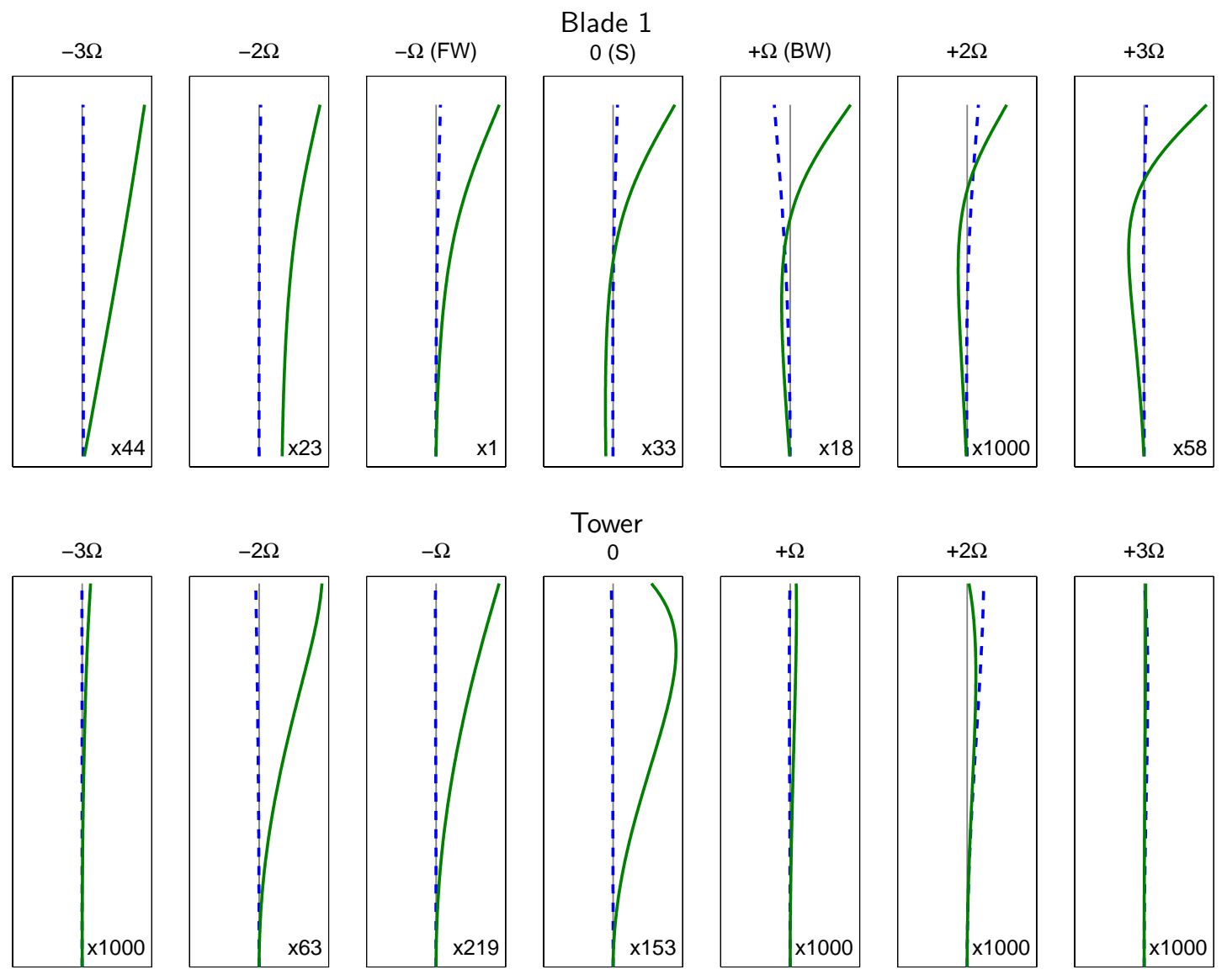

Figure 7: Amplitudes of harmonic components of the first flapwise forward whirling periodic mode shape for the anisotropic rotor with one blade covered with ice. Blade 1 (top) - flapwise, - - edgewise, and tower (bottom) - longitudinal and - - lateral.

system identification, is useful to check the validity of the linearisation.

The work presented in this paper is part of an ongoing effort to obtain a full aeroelastic linear model of the nonlinear code BHawC. The approach presented in this paper is readily extendable to a linear aeroelastic model. The linear model will aid in the understanding of the loads obtained from a nonlinear response, of which many features can be explained from the linear modes.

\section{Conclusion}

Tangent matrices for structural modal analysis are extracted directly from the nonlinear model of a wind turbine in a steady state. When the system is isotropic the preferred approach is to use the
Coleman transformation for describing the equations of motion in the inertial frame allowing direct eigenvalue analysis to extract the modal frequency, damping, and mode shapes. When the system is anisotropic, implicit Floquet analysis, that reduces the computational burden associated with classical Floquet analysis, is applied to yield the lowest damped eigenmodes. The linearised model is validated from numerical results for a three-bladed turbine, showing a reasonable agreement for the frequencies and the damping between the Coleman approach and partial Floquet analysis on the response of the nonlinear model for modes not related to the drivetrain. The implicit Floquet results converge to the results from the Coleman approach with the deviation in frequency and damping roughly proportional to the square of the integration time step and increasing with the modal frequency. This finding shows 

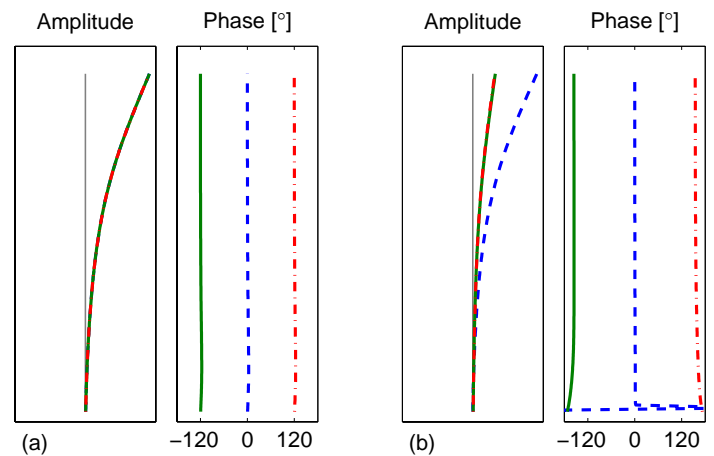

Figure 8: Amplitudes and phases of the harmonic component at $j=-1$ of the first flapwise forward whirling periodic mode shape for the isotropic rotor (a) and the anisotropic rotor (b). Blades - - 1 , -2 and.--3 .

the importance of precise time integration in implicit Floquet analysis. An analysis applied to an anisotropic system with one blade covered with ice shows a decrease in frequency up to $3 \%$ and changes in damping within $17 \%$. It also reveals multiple harmonic components in the response of a single mode which will show up in measurements.

\section{Acknowledgment}

This work has been partially supported by the Danish Ministry of Science, Technology and Innovation through the Industrial $\mathrm{PhD}$ programme.

\section{References}

[1] T.J. Larsen and A.M. Hansen. How 2 HAWC2, the user's manual. Technical Report Ris $\varnothing-$ R-1597(EN), Ris $\varnothing$ National Laboratory, Roskilde, Denmark, 2007.

[2] V.A. Riziotis and S.G. Voutsinas. Fatigue loads on wind turbines of different control strategies operating in complex terrain. Journal of Wind Engineering and Industrial Aerodynamics, 85(3):211-240, 2000.

[3] R. Rubak and J.T. Petersen. Monopile as part of aeroelastic wind turbine simulation code. In Proceedings of Copenhagen Offshore Wind, Denmark, October 2005.
[4] V.A. Riziotis, S.G. Voutsinas, E.S. Politis, and P.K. Chaviaropoulos. Aeroelastic stability of wind turbines: The problem, the methods and the issues. Wind Energy, 7(4):373392, 2004.

[5] T.G. van Engelen and H. Braam. TURBU offshore, computer program for frequency domain analysis of horizontal axis offshore wind turbines. Technical Report ECN-C-04079, Energy Research Centre of the Netherlands, 2004

[6] M.H. Hansen. Aeroelastic stability analysis of wind turbines using an eigenvalue approach. Wind Energy, 7:133-143, 2004.

[7] G. Bir and J. Jonkman. Aeroelastic instabilities of large offshore and onshore wind turbines. Journal of Physics: Conference Series, 75(1), 2007.

[8] B. Kirchgssner. ARLIS - a program system for aeroelastic analysis of rotating linear systems. In Proceedings of European Wind Energy Conference, pages 253-258, Hamburg, Germany, 1984.

[9] H.G. Matthies and C. Nath. Dynamic stability of periodic solutions of large scale nonlinear systems. Computer Methods in Applied Mechanics and Engineering, 48(2):191-202, 1985.

[10] K. Stol, M. Balas, and G. Bir. Floquet modal analysis of a teetered-rotor wind turbine. Journal of Solar Energy Engineering, 124:364-371, 2002.

[11] D.A. Peters. Fast Floquet theory and trim for multi-bladed rotorcraft. Journal of the American Helicopter Society, 39(4):82-89, 1994.

[12] O.A. Bauchau and Y.G. Nikishkov. An implicit Floquet analysis for rotorcraft stability evaluation. Journal of the American Helicopter Society, 46(3):200-209, 2001.

[13] K.A. Stol, H.-G. Moll, G. Bir, and H. Namik. A comparison of multi-blade coordinate transformation and direct periodic techniques for wind turbine control design. In Proceedings of 47th AIAA Aerospace Sciences Meeting, Orlando FL, USA, 2009. 
[14] G.H. James III, T.G. Carne, and J.P. Lauffer. The natural excitation technique (NExT) for modal parameter extraction from operating wind turbines. Report UC-261, Sandia National Laboratories, 1993.

[15] B. Marrant and Th. van Holten. System identification for the analysis of aeroelastic stability of wind turbine blades. In Proceedings of European Wind Energy Conference, London, UK, 2004.

[16] P.J. Murtagh and B. Basu. Identification of equivalent modal damping for a wind turbine at standstill using fourier and wavelet analysis. Proceedings of the Institution of Mechanical Engineers, Part K: Journal of Multibody Dynamics, 221(4):577-589, 2007.

[17] S. Krenk. Non-linear Modeling and Analysis of Solids and Structures. Cambridge University Press, Cambridge, 2009.

[18] W. Johnson. Helicopter Theory. Dover Publications, 1980.

[19] M.H. Hansen. Aeroelastic instability problems for wind turbines. Wind Energy, 10(6):551-577, 2007.

[20] M.H. Hansen. Improved modal dynamics of wind turbines to avoid stall-induced vibrations. Wind Energy, 6:179-195, 2003.

[21] L. Meirovitch. Methods of Analytical Dynamics. McGraw-Hill, New York, 1970.

[22] O.A. Bauchau and J. Wang. Efficient and robust approaches to the stability analysis of large multibody systems. Journal of Computational and Nonlinear Dynamics, 3, 2008.

[23] P.F. Skjoldan and M.H. Hansen. On the similarity of the Coleman and Lyapunov-Floquet transformations for modal analysis of bladed rotor structures. Journal of Sound and Vibration, 327:424-439, 2009.

[24] G.H. Golub and C.F. van Loan. Matrix Computations. The Johns Hopkins University Press, Baltimore, MD, 3rd edition, 1996.

[25] P.F. Skjoldan and O.A. Bauchau. Determination of modal parameters in complex nonlinear systems. Journal of Nonlinear and Computational Dynamics. Accepted for publication.
[26] Richtlinie fr Windenergieanlagen. Directive Reihe B, Heft 8, Deutsches Institut fr Bautechnik, Berlin, Germany, 2004.

[27] P.F. Skjoldan. Modal dynamics of wind turbines with anisotropic rotors. In Proceedings of 47th AIAA Aerospace Sciences Meeting, Orlando FL, USA, 2009. 